Knee Surg Sports Traumatol Arthrosc

DOI 10.1007/s00167-014-3160-9

\title{
Patellofemoral arthroplasty influences tibiofemoral kinematics: the effect of patellar thickness
}

Hilde Vandenneucker · Luc Labey · Jan Victor · Jos Vander Sloten · Kaat Desloovere · Johan Bellemans

H. Vandenneucker $(*) \cdot J$. Bellemans

Department of Orthopaedics, University Hospital Leuven, Weligerveld 1, 3212 PellenbergLubbeek, Belgium

e-mail: hilde.vandenneucker@uzleuven.be

L. Labey

European Centre for Knee Research, Smith and nephew, Technologielaan 11 bis, 3001 Leuven, Belgium

Biomechanics Section, University of Leuven, Celestijnenlaan 300c, 3001 Leuven, Belgium

J. Victor

Department of Orthopaedics and Traumatology, Ghent University Hospital, De Pintelaan 185, 9000 Ghent, Belgium

J. Vander Sloten

Biomechanics Section, University of Leuven, Celestijnenlaan 300c, 3001 Leuven, Belgium

K. Desloovere

Department of Rehabilitation Sciences, University Hospital Leuven, Weligerveld 1, 3212 Pellenberg-Lubbeek, Belgium 


\section{Abstract}

Purpose

Although controversy still remains, isolated patellofemoral arthroplasty recently gained in popularity as a treatment option for patellofemoral osteoarthritis. It has compared to total knee arthroplasty the advantage of preserving the tibiofemoral articulation, which in theory would allow the preservation of natural tibiofemoral kinematics. Today, however, no data exist to support this assumption. This study was therefore performed in order to investigate the effect of isolated patellofemoral arthroplasty on the native three-dimensional tibiofemoral kinematic behaviour and whether a change in patellar thickness would have an influence.

\section{Methods}

Six fresh-frozen cadavers were fixed on a custom- made mechanical knee rig. Full 3D kinematics was analysed during passive flexion-extension cycles, open chain extension, with and without mechanical resistance,as well as deep knee squats, using infrared motion capture cameras and retroflective markers. Measurements were taken for the native knee and after prosthetic trochlear resurfacing with and without patellar resurfacing in three different patellar thicknesses.

\section{Results}

Compared to the natural knee, patellofemoral arthroplasty resulted in significant changes in tibiofemoral kinematics, which were most pronounced in the most loaded motor tasks. Increased internal tibial rotation was noted in the mid- and high flexion ranges, reaching at $120^{\circ}$ of flexion a mean difference of $4.5^{\circ} \pm 4.3^{\circ}(p<0.0001)$ during squat motion, over the whole flexion range during open chain motion and in deeper flexion beyond $50^{\circ}$ (mean at $70^{\circ}, 1.9^{\circ} \pm$ $3.7^{\circ}$ ) during resisted open chain. During squats, also, a more posterior translation of the lateral femoral condyle was observed. The effect was accentuated in case of patella overstuffing, whereas kinematics was closer to normal with patellar thinning.

\section{Conclusion}

Isolated patellofemoral arthroplasty alters natural tibiofemoral kinematics, and the effects become more pronounced in case of increased patellar thickness. Therefore, it might be recommended to aim for a slight over-resection of patellar bone if sufficient bone stock is available. 


\section{Keywords}

PFA - Tibiofemoral kinematics $\cdot$ Patella resurfacing $\cdot$ Overstuffing 


\section{Introduction}

While isolated patellofemoral arthritis might not be as prevalent as patellofemoral involvement in the general degenerative knee [6], it nevertheless has been reported in $8 \%$ of women over the age of 55 years. Although total knee arthroplasty (TKA) has been proven to be a successful option in treating these patients $[1,3-5,8-13,15,16,22]$, their relatively young age has driven the interest towards a more conservative treatment option.

The early resurfacing inlay patellofemoral designs simply replaced the damaged trochlear cartilage and reproduced the shape of the native subchondral bone. The curvature and width of the implant did not reproduce the normal patellar tracking, leading to a high percentage of persisting anterior knee pain and snapping problems [14].

The second generation, so-called anterior cut, patellofemoral prostheses (PFP) are based on the TKA design and replace the entire anterior compartment, with a broad trochlear surface and a more proximal lateral flange $[2,14]$. Both symmetrical and asymmetrical prostheses, with a valgus tracking angle, were developed. This resulted in less mechanical catching problems and a better patellar kinematic behaviour. Since the predominant reason for failure of the current generation patellofemoral implants is the progression of disease in the medial tibiofemoral compartment [21], the focus has been re-orientated from design issues $[2,24]$ towards indication and patient selection $[11,20,28]$.

The claimed advantages of patellofemoral arthroplasty (PFA) include conservation of bone stock, faster and easier revalidation and reproduction of a more natural general kinematic behaviour of the knee. As the tibiofemoral articular surfaces, ligaments and menisci are not altered, the tibiofemoral kinematics is believed to be maintained. However, there is currently a lack of biomechanical studies supporting this assumption. The available literature involving PFA is indeed mainly focused upon clinical results, complications and survivorship $[13,14]$.

The purpose of this study was therefore to compare the three-dimensional tibiofemoral kinematics of a contemporary patellofemoral design (Journey PFJ, Smith and Nephew, TN, USA) with those of the natural knee, using a previously developed and slightly adapted in vitro model for kinematic knee analysis [26, 27], and to investigate the influence of patellar over- and understuffing. The hypothesis was that a well-placed and oriented PFP would not alter tibiofemoral kinematics. This should make PFA more appealing as an alternative treatment option for isolated patellofemoral arthritis, as the currently available TKA designs do not fully reproduce the natural tibiofemoral kinematics. 


\section{Materials and methods}

Six unmatched lower leg specimens, from Caucasian subjects (one female, five males, mean age 80.5 years, range $78-91$ years), were disarticulated at the hip and frozen at $-20^{\circ} \mathrm{C}$. The tested specimens had no signs of previous surgery, traumatic bone or ligament lesions. none of the specimens had major arthritic damage at the level of the knee. Three frames, with on each frame four reflective marker spheres (NDI, Waterloo, Ontario, Canada), were rigidly fixed to the frozen specimens: one at the level of the femoral neck, one in the proximal tibia at a minimum of $10 \mathrm{~cm}$ distal to the joint and one onto the anterior aspect of the patella. A volumetric CT scan was performed (Siemens Somatom Definition Flash, Siemens AG, Erlangen, Germany). The images were obtained at $120 \mathrm{kV}$ and $200 \mathrm{mAs}+$ Care Dose, with a pitch of $0.8 \mathrm{~mm}$ per revolution, $1 \mathrm{~mm}$ slice thickness and slice increment, $1.0 \mathrm{~s}$ rotation time and B70-B30 reconstruction kernel.

Each specimen was thawed during $36 \mathrm{~h}$ before the start of the experiments. The threedimensional motion capture system, composed of six infrared cameras (Vicon Motion Systems, LA, California), was positioned in such way that the optical reference markers on patella, tibia and femur were visible, both with the specimen lying on the operating table, and with the specimen mounted in the knee rig. The system was calibrated before starting each cadaver test. After removing the skin, a second marker frame was fixed to the femoral diaphysis and the spatial relation between the two femoral frames was measured with the camera system. The proximal part of the femur was then amputated $32 \mathrm{~cm}$ proximal to the knee joint. The foot was amputated $28 \mathrm{~cm}$ distal to the knee joint, and the soft tissues at the free ends of femur and tibia were removed over a distance of $12 \mathrm{~cm}$. Both free ends were then embedded in metal containers with PMMA, taking care of the physiologic alignment of femur and tibia. The quadriceps tendon was prepared and rigidly fixed in a clamp at a distance of $6 \mathrm{~cm}$ above the proximal patellar pole. The other end of the clamp was fixed to a servomotor by means of a ball bearing so that it could rotate freely in all directions, thus allowing proper tracking of the patella in the trochlear groove. On the lateral side, the biceps tendon was isolated and sutured with number 2 Vicryl. In the same way, the semitendinosus and semimembranosus tendon were prepared at the medial side.

In a next step, the prepared specimen was mounted on the customized dynamic knee rig, simulating normal knee motions and loads and leaving six degrees of freedom [27]. One actuator simulated the quadriceps muscle, another produced vertical hip motion. Medial and 
lateral hamstrings were loaded $(50 \mathrm{~N})$ by attaching them to two constant load springs in a position simulating the natural in vivo moment arms [25]. Sensors measured quadriceps and ankle forces and moments, as well as the hip height relative to the ankle. The hip joint allowed flexion-extension and could move up and down, and the ankle joint allowed medio-lateral translation and all rotational movements.

Each specimen was tested in 4 motor tasks (Fig. 1): passive motion, an open chain movement without resistance and with 3-kg resistance at the ankle, and a deep knee squat. Passive motion was induced manually with three cycles from extension to maximal flexion, with the femoral container mounted on the rig. The three other motor tasks were performed while loading quadriceps and medial and lateral hamstrings. During the open chain movement, the leg was extended at a fixed speed from about $110^{\circ}$ of flexion to about $-20^{\circ}$ of extension by pulling on the quadriceps tendon with variable load, while the ankle was hanging free. Near full extension, the quadriceps load reached values between 60 and $100 \mathrm{~N}$, depending on the weight of the lower leg. The resisted open chain was performed in the same way, with a $3 \mathrm{~kg}$ weight fixed to the tibial container at the distal end. Close to full extension quadriceps load reached values around $300 \mathrm{~N}$. For the squat, the tibial container was also mounted in the rig. The hip height was programmed as a function of time, thus controlling flexion of the knee between $20^{\circ}$ and $120^{\circ}$ of flexion, while applying a variable quadriceps force to induce a vertical constant ankle load of $130 \mathrm{~N}$. During squatting, the quadriceps load was highest at maximal flexion with values around $1,000 \mathrm{~N}$.

The six infrared cameras dynamically registered the motion of the marker frames on femur, tibia and patella as a function of time. Meanwhile, calibrated load cells recorded the loads on the quadriceps tendon and the ankle. A second post-test CT was then performed to confirm the unchanged positions of the reference frames.

In a first step, the natural knee was tested, as described above. This was done after opening the medial retinaculum and then closing it again, without further touching the joint. Thus, we measured tibiofemoral kinematic reference data with the medial retinaculum in a situation similar to the situation after PFA. Consequently, any measured difference between the reference data and the kinematics after PFA was considered to be due to changes induced by the PFA. In a second step, a trochlear resurfacing was performed, using the trochlear component of the Journey PF prosthesis (Smith-Nephew, Memphis, TN, USA), without resurfacing the patella. All measurements were repeated again. In the next steps, patella 
resurfacing was performed, using a biconvex button centred on the ridge, starting with a 3-mm under-resection (overstuffing), followed by a reconstruction of the natural patellar thickness and by a 3-mm over-resection (thinning). After each step, the joint was closed and the knee was tested while performing the four different motor tasks.

\section{Data processing}

The pretest CT data were loaded and analysed using Mimics 11.02 and its MedCAD module (Materialise, Haasrode, Belgium). Surface reconstructions of femur, tibia and patella were created, and the relevant bony landmarks were identified, starting from the quantitative morphological description by LaPrade et al., and as defined, with description of intra- and interobserver variability, in the previous work of Victor [26]. Based on these landmarks, relevant axes and planes on tibia and femur were determined. The motions of the bones were digitally reconstructed. CT data and motion capture data were combined which allowed the reconstruction of the anatomic landmarks during motion trials. Anatomic bone coordinate systems and joint rotations were calculated based on the Grood and Suntay knee model. However, more accurate femoral landmarks were used [27]. To define internal-external rotation of the femoral frontal plane, the line joining the medial and lateral condyle centres was used. Additional measurements were taken, including the antero-posterior translations of the medial (MFCC) and lateral femoral condyle centres (LFCC), projected onto the tibial horizontal plane. The translations were measured as the perpendicular distance of each condyle centre to the line connecting the MFCC and LFCC, also projected onto the tibial horizontal plane.

Measurements of tibiofemoral kinematics included (internal/external) rotation of the tibia around its own long axis with respect to the femur, and anterior-posterior (AP) translations of MFCC and LFCC. The data were presented as a function of tibiofemoral flexion angle, every $10^{\circ}$, from $0^{\circ}$ to $130^{\circ}$ for passive motion, from $0^{\circ}$ to $110^{\circ}$ for open chain movements, and from $30^{\circ}$ to $120^{\circ}$ for squats. Accuracy and precision of the motion analysis system, used for the kinematic recordings of the markers, was on the order of 0.2-0.3 $\mathrm{mm}$. Previous repeatability calculations revealed that $95 \%$ of the measurements would be expected to fall within the range of $0.5^{\circ}$ for tibial rotations, $0.8 \mathrm{~mm}$ for MFCC translations and $0.8 \mathrm{~mm}$ for LFCC translations. The translations were, however, expressed as a fraction of the AP size of the tibia, from the anterior cortical bone of the tibial tubercle to the posterior border of the tibial bone, in order to compensate for differences in AP tibial sizes of the specimens.

This study was approved by the ethical committee of the University Hospitals KU Leuven. 


\section{Statistical analysis}

Linear mixed models were used to analyse the differences between the four settings. Random effects account for correlation between repeated observations within the same specimen. The models include a random intercept for specimen (general or setting specific) and a random slope for flexion angle. The evolution of motion over an increasing flexion angle is modelled with linear, quadratic or splinesbased models. The model with the best fit lowest Akaike information criterion (AIC) was selected and used for inference. In a first step, the interaction between setting and flexion angle was tested using a likelihood ratio test. In case of significant interaction, pairwise differences between settings were analysed at three different flexion angles $\left(30^{\circ}-70^{\circ}-110^{\circ}\right)$. In case of non-significant interaction, a likelihood ratio test was performed for a main effect of setting. In case of an overall effect, all pairwise differences between settings were further tested. Bonferroni step-down correction for multiple testing was performed. A $5 \%$ significance level was assumed. All analyses were performed using the SAS package, version 9.2 of the SAS System for Windows.

\section{Results}

Squat (Fig. 2; Tables 1, 2)

From $30^{\circ}$ to $70^{\circ}$, the tibia of the natural knee rotated on average $5.9^{\circ} \pm 2.7^{\circ}$ internally (range $3.5^{\circ}-8.8^{\circ}$ ), followed by a much slower further internal rotation over on average $2.3^{\circ} \pm 1.5^{\circ}$ (range $0.8^{\circ}-3.8^{\circ}$ ) up to $120^{\circ}$ of flexion. Over the whole range of motion (from $30^{\circ}$ to $120^{\circ}$ ), a mean internal tibia rotation of $8.8^{\circ} \pm 1.1^{\circ}$ (range $8.1^{\circ}-9.6^{\circ}$ ) is observed.

Isolated trochlear resurfacing did not cause any significant change in tibial internal rotation. However, additional patella resurfacing, with restoration of the original patellar thickness, led to significantly more internal rotation compared to the natural joint in the deeper flexion range. A 3-mm underresection (or overstuffing) of the patella accentuated this increase in tibial internal rotation. There was a significant difference $(p<0.0001)$ in slope of the curve with a faster increase in tibial internal rotation as a function of the knee flexion when the patella was under-resected. Thinning the patella by $3 \mathrm{~mm}$ had the opposite effect.

For the translations, no differences were observed in translation of the MFCC. For every degree of knee flexion, the MFCC was moving posteriorly over $1 / 1,000$ th (95 \% Cl 0.0015; 0.0005) of the tibial AP width. The LFCC translation was the same for the natural knee and the knee with isolated trochlea resurfacing, giving a small posterior translation over $2 / 1,000$ th $(95 \% \mathrm{Cl} 0.002$; 
0.001) of the tibial AP width. neutral patella resurfacing resulted in deep flexion in more posterior translation of the LFCC, which was significantly less pronounced with a 3-mm overresection of the patellar bone.

Open chain with resistance (Fig. 3; Tables 3, 4)

In the resisted open chain motion, trochlear resurfacing resulted in a less internally rotated tibia in low and midflexion, compared to the natural knee, which showed a constant rotational position. In deeper flexion, the natural knee showed some external rotation, while the trochlear resurfaced knee kept almost the same rotation. After resurfacing the patella and restoring its natural height, more tibial internal rotation was measured in the deeper flexion range. Changing the patellar height did not give consistent findings. There were no consistent differences observed in femoral condyle translation.

\section{Open chain without resistance}

In the non-resisted open chain motion, no differences were observed between the natural joint and the trochlea resurfacing concerning tibial rotation, but patella resurfacing again resulted in significant more internal tibial rotation over the whole flexion range $(p<0.01)$. no differences were seen in translation of the femoral condyle centres.

\section{Passive motion}

In general, the tibia rotated on average $12.6^{\circ} \pm 2.8^{\circ}$ internally (range $8.6^{\circ}-15.1^{\circ}$ ) with increasing flexion between $0^{\circ}$ and $60^{\circ}$, without any further rotation in deeper flexion. In deep flexion, more internal tibial rotation was noted after trochlea resurfacing, both with and without patella resurfacing, regardless of the thickness of the patella $(p<0.01)$. In general, the LFCC translated posteriorly with increasing flexion beyond a flexion of $70^{\circ}$, up to $120^{\circ}$, over $2 / 10$ th of the tibial AP width $(0.21 \pm 0.04)$, whereas the MFCC was only showing a posterior translation in this range of motion over $1 / 10$ th $(0.1 \pm 0.04)$ of the AP width. Overall no differences were observed in FCC translation between the different conditions.

\section{Discussion}

The most important finding of the present study was that a standard PFA with patella resurfacing, reconstructing the natural patellar thickness, induced changes in tibiofemoral knee kinematics which were most pronounced in the most loaded motor tasks. Based on these results, our hypothesis is rejected. Isolated trochlear resurfacing did not induce significant changes, compared to the natural situation, in tibial rotation and translation of the femoral 
condyle centres, when performing a squat or a non-resisted open chain motion. However, additional neutral patella resurfacing resulted, in the mid- and high flexion ranges of a squat, in more internal tibial rotation and a more posterior translation of the LFCC. When performing a resisted open chain motion, isolated trochlea resurfacing revealed in the low and mid-flexion ranges a less internal rotated tibia compared to the normal situation. With resurfacing the patella similar, more internal tibial rotation was measured in the higher flexion ranges, as observed within a non-resisted open chain. Again, there were no significant differences in translation. In the non-resisted open chain, more internal tibial rotation was noted over the whole flexion range, without differences in sagittal femoral translation. In passive motions, overall more internal tibial rotation was noted in the low flexion angles when the trochlea is resurfaced, regardless of patella resurfacing.

Patella overstuffing accentuated the observed changes, at least when performing a squat movement. Likewise, patellar over-resection diminished the observed changes and restored tibiofemoral kinematics more close to the natural situation.

These findings might be explained by the fact that trochlear resurfacing is creating an overstuffing of the joint, both due to geometrical changes and to a difference in compressibility of the metal of the component as compared to articular cartilage. This specific design has al lateralized trochlear groove with a higher lateral flange. When the patella is not resurfaced, the compressible natural cartilage of the knee cap can compensate for the above mentioned differences to some extent, which explains the preservation of the natural tibiofemoral kinematics. Placing a hard polyethylene button on the articular patellar surface eliminates this effect and therefore results in relative overstuffing of the patellofemoral joint, even though the original patellar thickness was maintained. Three millimetre of extra patellar resection reduces the initial thickness of the anterior compartment, and the previously observed changes in tibiofemoral kinematics become therefore less obvious. The same reasoning was proposed in a study of Merican et al. [17] concerning the influence of patellar thickness on patellofemoral kinematics in TKA. They also contributed kinematic changes, observed when restoring the native patellar thickness, to an anterior overstuffing by increased thickness of the anterior prosthetic flange. However, they did not observe a change in tibial rotation of the TKA with building up the anterior joint.

Since the tibiofemoral joint is not modified in PFA, the observed tibiofemoral kinematic changes in our study have to be secondary to alterations in the patellofemoral joint. Patellofemoral 
kinematics was also measured. However, in general, natural patellofemoral kinematics was much bet- ter reproduced when the patella was resurfaced, which is in contrast with the observed changes in tibiofemoral kinematics after patella resurfacing. Whereas without patella resurfacing a significant $(p<0.0001)$ higher lateral patellar rotation and tilt were measured in mid-flexion, there were no significant changes in patellar rotation, tilt or medial- lateral translation after patella resurfacing, compared to the natural situation. neutral patella resurfacing only resulted in a more anterior patellar position, conforming our assumption that the observed tibiofemoral kinematic changes are induced by a relative overstuffing of the anterior compartment.

Building up the anterior compartment of the knee can be expected to result in a more posterior position of the FCC on the tibial plateau. If, as we presume, trochlear resurfacing is creating a general overstuffing of the patellofemoral joint in this type of PFA, a change in sagittal plane tibiofemoral kinematics can be explained. This is comparable with the results of Monk et al. [19] who measured sagittal plane kinematics after PFA, using the FPV implant (Wright Medical Technology, Inc, Arlington, Tenn), in an in vivo fluoroscopic study, performing a step-up exercise and a lunge exercise. They found no differences in patellofemoral tendon angle (PTA) between the natural knees and the PFA group during step- ups, but measured a mean lower PTA in the prosthetic group when performing a lung exercise, with differences being significant in the flexion range from $90^{\circ}$ to $130^{\circ}$, which is consistent with our findings of a more posteriorly positioned LFCC during squat exercises. However, they attributed the lower PTA to a deeper position of the patella in the trochlea and therefore to an under-stuffing, either patellar, or trochlear, of the joint. As an extra patellar resection and thus thinner patella in our measurements is resulting in the opposite effect, this explanation does not seem to be correct. In our opinion, the lower PTA has to be explained by a more posterior position of the femur and consequently a more posterior position of the patella with respect to the tibia, creating a lower PTA. Our measurements of the patellofemoral kinematics after PFA with patella resurfacing in a separate study show a clearly more anterior position of the patella relative to the femur and are an argument against the cantilever theory of Monk [18] as an explanation for the 'deeper' patella position in the trochlea. The PTA is not in any way directly related to the threedimensional position of the femur, as it is defined as the angle between the long axis of the tibia and the line along the length of the patellar tendon. It is giving a relationship between the position of the tibia and the position of the patella and a lower PTA therefore only means a 
deeper position in the trochlea when the femoral position is stable, which is not the case in squat motions after PFA. In a previous, similar study [7] on the in vivo sagittal plane kinematics of the Avon PFA (Stryker, newbury, UK), the same group of investigators found a consistent higher PTA in the prosthetic group, which was thought to be caused by the surface geometry of the prosthesis. Stagni et al. [23] also concluded in their study that the PTA/KFA relationship cannot be used as a quantitative indicator of the antero-posterior translation of the femur over the tibia.

As the thickness of the patella was meticulously reproduced to the natural values in our study, the overstuffing of the anterior joint has to be attributed to a more pronounced trochlea, at least in loaded conditions.

Rotational tibial alignment was not measured in Monk's study [18]. To our knowledge, no study has been published so far measuring the tibial rotation after patellofemoral arthroplasty. The consistently higher tibial internal rotation during all motor tasks, at least in the mid- and high flexion angles, can, in our opinion, also be explained by the overstuffing of the anterior joint. As the medial tibial condyle is more stable than the lateral, the anterior translation of the lateral tibial condyle, caused by anterior overstuffing, will be more pronounced, which consequently induces more tibial internal rotation. The natural tibiofemoral rotational motion, induced by the cruciate ligaments and the joint congruence, is indeed additionally guided by the extensor mechanism. The $Q$ angle reduces with increasing flexion. This leads the patella to a more medial position in the trochlear groove, which consequently helps to steer the tibial internal rotation. Increasing anterior stuffing will further accentuate this effect.

One could also argue that an alteration in trochlear rotation, induced by the trochlear resurfacing procedure, is a possible cause of the change in tibial rotation. A more externally rotated femoral component will induce more internal tibial rotation during flexion of the knee. Correlating the degree of femoral component rotation with the difference in tibial rotation in our study indeed confirmed the fact that the most externally rotated trochlear components induced the highest tibial internal rotation. As the average trochlear component rotation in our study was $5.3^{\circ}$ internal rotation (SD 3.61), measured as the rotation of the anterior cutting surface relative to the epicondylar axis, this cannot, however, explain the higher tibial internal rotation after PFA compared to the natural knee in our study.

This study has a number of limitations to be considered. It was a cadaver study with a limited number of specimens and performed by a single surgeon. The trochlear components were 
placed without navigation control and seemed to be positioned somewhat in internal rotation. As femoral endorotation induces more tibial external rotation, more external rotated trochlear components are expected to induce an even bigger increase in tibial internal rotation than the increase we measured in our study. The order of testing might have had an effect on the results as well, although there is no alternative for the order in which the tests were performed. The study analysed the result of a specific prosthetic design (Journey PFJ S\&n). nevertheless, as the stiffness of the prosthetic material is comparable for all types of design, we believe that our findings can be extrapolated to all PFP of the second generation. While the measured motions simulate actual motor tasks, they are not representative of daily life situations. Changes in tibiofemoral kinematics during level walking were for example not measured. And finally, the ITB was not loaded and is known to induce more tibial external rotation [18]. However, as this study compares the kinematic behaviour between the natural knee and the PFA in the same unloaded ITB conditions, we believe that this should have no major influence on our findings. As the observed changes in tibiofemoral kinematics after performing a standard PFA are probably due to an overstuffing of the patellofemoral joint and as natural kinematic behaviour is more closely reproduced when the patella is slightly over-resected, the findings have a clinical relevance with respect to all isolated patellofemoral arthroplasties. If sufficient patellar bone stock is available, it might be recommended to resect some additional patellar bone in order to thin the patella in a classical patellofemoral arthroplasty and to avoid in this way the effect of overstuffing.

\section{Conclusion}

Our results are not supporting the claim that a standard patellofemoral arthroplasty, with restoration of the original patellar thickness, maintains the normal tibiofemoral kinematics. More internal tibial rotation and more posterior translation of the LFCC could be observed. A small additional resection of patellar bone resulted in a closer reproduction of normal kinematics and might therefore be advisable.

\section{Acknowledgments}

This study was performed in the European Centre for Knee Research of Smith and nephew. The authors want to thank Ronny Decorte and Bernardo Innocenti for their continuous practical support. 


\section{Conflict of interest}

Some of the authors have a sporadic consultancy agreement (lectures) with Smith and nephew.

\section{References}

1. Ackroyd Ce, newman JH, evans R, eldridge JD, Joslin CC (2007) The Avon patellofemoral arthroplasty: fiveyear survivorship and functional results. J Bone Joint Surg $\mathrm{Br}$ 89(3):310-315

2. Amis AA, Senavongse W, Darcy P (2005) Biomechanics of patellofemoral joint prostheses. Clin Orthop Relat Res 436:20-29

3. Argenson Jn, Flecher X, Parratte S, Aubaniac JM (2005) Patellofemoral arthroplasty: an update. Clin Orthop Relat Res 440:50-53

4. Beitzel K, Schottle PB, Cotic M, Dharmesh V, Imhoff AB (2013) Prospective clinical and radiological twoyear results after patellofemoral arthroplasty using an implant with an asymmetric trochlea design. Knee Surg Sports Traumatol Arthrosc 21(2):332-339

5. Cartier P, Sanouiller JL, Khefacha A (2005) Long-term results with the first patellofemoral prosthesis. Clin Orthop Relat Res 436:47-54

6. Donell ST, Glasgow MM (2007) Isolated patellofemoral osteoarthritis. Knee 14(3):169-176

7. Hollinghurst D, Stoney J, Ward T, Pandit H, Beard D, Murray DW (2007) In vivo sagittal plane kinematics of the Avon patellofemoral arthroplasty. J Arthroplasty 22(1):117-123

8. Karlsson J (2012) The forgotten joint. Knee Surg Sports Trauma- tol Arthrosc 20(7):1215

9. Kooijman HJ, Driessen AP, van Horn JR (2003) Long-term results of patellofemoral arthroplasty. A report of 56 arthroplasties with 17 years of follow-up. J Bone Joint Surg Br 85(6):836-840

10. Krajca-Radcliffe JB, Coker TP (1996) Patellofemoral arthroplasty. A 2- to 18-year followup study. Clin Orthop Relat Res 330:143-151

11. Leadbetter WB, Seyler TM, Ragland PS, Mont MA (2006) Indications, contraindications, and pitfalls of patellofemoral arthroplasty. J Bone Joint Surg Am 88(Suppl 4):122-137

12. Lonner JH (2007) Patellofemoral arthroplasty. J Am Acad Orthop Surg 15(8):495-506

13. Lotke PA, Lonner JH, nelson CL (2005) Patellofemoral arthroplasty: the third compartment. J Arthroplasty 20(4 Suppl 2):4-6

14. Lustig S, Magnussen RA, Dahm DL, Parker D (2012) Patellofemoral arthroplasty, where are we today? Knee Surg Sports Traumatol Arthrosc 20(7):1216-1226

15. Meding JB, Wing JT, Keating eM, Ritter MA (2007) Total knee arthroplasty for isolated patellofemoral arthritis in younger patients. Clin Orthop Relat Res 464:78-82

16. Merchant AC (2004) early results with a total patellofemoral joint replacement arthroplasty prosthesis. J Arthroplasty 19(7):829-836 
17. Merican AM, Ghosh KM, Baena FR, Deehan DJ, Amis AA (2012) Patellar thickness and lateral retinacular release affects patellofemoral kinematics in total knee arthroplasty. Knee Surg Sports Traumatol Arthrosc 22(3):526-533

18. Merican AM, Amis AA (2009) Iliotibial band tension affects patellofemoral and tibiofemoral kinematics. J Orthop Res 27(3):335-339

19. Monk AP, van Duren BH, Pandit H, Shakespeare D, Murray DW, Gill HS (2012) In vivo sagittal plane kinematics of the FPV patellofemoral replacement. Knee Surg Sports Traumatol Arthrosc 20(6):1104-1109

20. Mont MA, Johnson AJ, naziri Q, Kolisek FR, Leadbetter WB (2012) Patellofemoral arthroplasty: 7-year mean follow-up. J Arthroplasty 27(3):358-361

21. nicol SG, Loveridge JM, Weale Ae, Ackroyd Ce, newman JH (2006) Arthritis progression after patellofemoral joint replacement. Knee 13(4):290-295

22. Smith AM, Peckett WR, Butler-Manuel PA, Venu KM, d'Arcy JC (2002) Treatment of patello-femoral arthritis using the Lubinus patello-femoral arthroplasty: a retrospective review. Knee 9(1):27-30

23. Stagni R, Fantozzi S, Catani F, Leardini A (2010) Can Patellar Tendon Angle reveal sagittal kinematics in total knee arthroplasty? Knee Surg Sports Traumatol Arthrosc 18(7):949-954

24. Stiehl JB (2005) A clinical overview patellofemoral joint and application to total knee arthroplasty. J Biomech 38(2):209-214

25. Victor J, Labey L, Wong P, Innocenti B, Bellemans J (2010) The influence of muscle load on tibiofemoral knee kinematics. J Orthop Res 28(4):419-428

26. Victor J, Van Doninck D, Labey L, Innocenti B, Parizel PM, Bellemans J (2009) How precise can bony landmarks be determined on a CT scan of the knee? Knee 16(5):358-365

27. Victor J, Van Glabbeek F, Vander Sloten J, Parizel PM, Somville J, Bellemans J (2009) An experimental model for kinematic analysis of the knee. J Bone Joint Surg Am 91(Suppl 6):150-163

28. Walker T, Perkinson B, Mihalko WM (2012) Patellofemoral arthroplasty: the other unicompartmental knee replacement. J Bone Joint Surg Am 94(18):1712-1720 
Table 1 Mean (SD) tibial rotation for squat motions in the native knee and differences in tibial rotation in resurfaced conditions compared to the native knee, at different tibiofemoral flexion angles

\begin{tabular}{|c|c|c|c|c|c|c|c|c|c|c|c|c|c|c|}
\hline \multirow{3}{*}{$\begin{array}{l}\text { Tibiofemoral } \\
\text { flexion }\end{array}$} & \multirow{2}{*}{\multicolumn{2}{|c|}{ Native opened }} & \multicolumn{12}{|c|}{ Difference wrt. native opened } \\
\hline & & & \multicolumn{3}{|l|}{ PFJ } & \multicolumn{3}{|c|}{ PFJ + P neutral } & \multicolumn{3}{|c|}{$\mathrm{PFJ}+\mathrm{P}$ under } & \multicolumn{3}{|c|}{$\mathrm{PFJ}+\mathrm{P}$ over } \\
\hline & Mean & SD & Mean & SD & & Mean & $\mathrm{SD}$ & & Mean & $\mathrm{SD}$ & & Mean & $\mathrm{SD}$ & \\
\hline 30 & -0.2 & 2.0 & 0.0 & 4.7 & ns & 0.3 & 2.4 & ns & 0.3 & 4.0 & ns & -0.1 & 3.2 & ns \\
\hline 40 & -2.9 & 2.7 & -0.8 & 3.9 & ns & 0.2 & 2.2 & ns & 0.1 & 3.5 & ns & -0.2 & 3.0 & ns \\
\hline 50 & -4.3 & 3.5 & -1.1 & 3.8 & ns & -0.1 & 2.4 & ns & 0.3 & 3.1 & ns & -0.1 & 3.0 & ns \\
\hline 60 & -5.2 & 4.1 & -1.0 & 3.6 & ns & -0.0 & 2.3 & ns & 0.7 & 2.8 & $* *$ & -0.2 & 2.6 & ns \\
\hline 70 & -5.8 & 4.0 & -1.0 & 3.2 & ns & 0.3 & 2.2 & $* * *$ & 1.1 & 2.6 & $* * *$ & -0.0 & 2.5 & $*$ \\
\hline 80 & -5.9 & 4.5 & -1.0 & 2.7 & ns & 1.3 & 1.6 & $* * *$ & 2.2 & 2.3 & $* * *$ & 0.8 & 2.0 & $* * *$ \\
\hline 90 & -6.5 & 4.1 & -0.4 & 1.9 & ns & 2.4 & 1.4 & $* * *$ & 3.3 & 1.7 & $* * *$ & 1.6 & 1.4 & $* * *$ \\
\hline 100 & -6.8 & 4.2 & 0.0 & 1.3 & ns & 3.2 & 2.4 & $* * *$ & 4.4 & 2.8 & $* * *$ & 2.0 & 2.4 & $* * *$ \\
\hline 110 & -6.9 & 3.8 & 0.4 & 0.9 & ns & 4.4 & 2.8 & $* * *$ & 6.0 & 3.1 & $* * *$ & 3.0 & 2.7 & $* * *$ \\
\hline 120 & -8.2 & 2.0 & 0.9 & 0.6 & ns & 4.5 & 4.3 & $* * *$ & 5.2 & 4.0 & $* * *$ & 3.9 & 3.5 & $* * *$ \\
\hline
\end{tabular}

$n s$ Not significant

$* p<0.05 ; * * p<0.001 ; * * * p<0.0001$

Table 2 Mean (SD) LFCC translation for squat motions in the native knee and differences in translation in resurfaced conditions compared to the native knee, at different flexion angles

\begin{tabular}{|c|c|c|c|c|c|c|c|c|c|c|c|c|c|c|}
\hline \multirow{3}{*}{$\begin{array}{l}\text { Tibiofemoral } \\
\text { flexion }\end{array}$} & \multirow{2}{*}{\multicolumn{2}{|c|}{ Native opened }} & \multicolumn{12}{|c|}{ Difference wrt. native opened } \\
\hline & & & \multicolumn{3}{|l|}{ PFJ } & \multicolumn{3}{|c|}{$\mathrm{PFJ}+\mathrm{P}$ neutral } & \multicolumn{3}{|c|}{$\mathrm{PFJ}+\mathrm{P}$ under } & \multicolumn{3}{|c|}{$\mathrm{PFJ}+\mathrm{P}$ over } \\
\hline & Mean & SD & Mean & SD & & Mean & SD & & Mean & SD & & Mean & SD & \\
\hline 30 & -0.690 & 0.039 & 0.002 & 0.042 & ns & 0.002 & 0.020 & * & 0.003 & 0.046 & ns & 0.004 & 0.030 & ns \\
\hline 40 & -0.707 & 0.036 & -0.007 & 0.038 & ns & -0.001 & 0.018 & ns & 0.006 & 0.042 & $\mathrm{~ns}$ & 0.001 & 0.033 & ns \\
\hline 50 & -0.734 & 0.040 & -0.009 & 0.026 & ns & -0.009 & 0.019 & $\mathrm{~ns}$ & 0.007 & 0.028 & ns & -0.001 & 0.027 & $\mathrm{~ns}$ \\
\hline 60 & -0.745 & 0.033 & -0.011 & 0.022 & * & -0.008 & 0.018 & ns & 0.010 & 0.024 & * & -0.005 & 0.027 & $\mathrm{~ns}$ \\
\hline 70 & -0.763 & 0.035 & -0.016 & 0.024 & $*$ & -0.007 & 0.020 & ns & 0.009 & 0.020 & $* * *$ & -0.004 & 0.031 & $\mathrm{~ns}$ \\
\hline 80 & -0.779 & 0.034 & -0.013 & 0.029 & ns & 0.009 & 0.013 & $* * *$ & 0.023 & 0.017 & $* * *$ & 0.002 & 0.019 & ns \\
\hline 90 & -0.794 & 0.023 & -0.007 & 0.029 & ns & 0.018 & 0.017 & $* * *$ & 0.031 & 0.024 & $* * *$ & 0.008 & 0.014 & ns \\
\hline 100 & -0.801 & 0.028 & -0.003 & 0.007 & ns & 0.034 & 0.030 & $* * *$ & 0.050 & 0.050 & $* * *$ & 0.011 & 0.033 & $*$ \\
\hline 110 & -0.820 & 0.014 & 0.001 & 0.007 & ns & 0.051 & 0.038 & **** & 0.073 & 0.060 & $* * *$ & 0.028 & 0.030 & * \\
\hline 120 & -0.838 & 0.016 & 0.005 & 0.012 & ns & 0.041 & 0.047 & $* * *$ & 0.050 & 0.064 & $* * *$ & 0.025 & 0.030 & $*$ \\
\hline
\end{tabular}


Table 3 Mean (SD) tibial rotation for resisted open chain motions in the native knee and differences in tibial rotation in resurfaced conditions compared to the native knee, at different flexion angles

\begin{tabular}{|c|c|c|c|c|c|c|c|c|c|c|c|c|c|c|}
\hline \multirow{3}{*}{$\begin{array}{l}\text { Tibiofemoral } \\
\text { flexion }\end{array}$} & \multirow{2}{*}{\multicolumn{2}{|c|}{ Native opened }} & \multicolumn{12}{|c|}{ Difference wrt. native opened } \\
\hline & & & \multicolumn{3}{|l|}{ PFJ } & \multicolumn{3}{|c|}{$\mathrm{PFJ}+\mathrm{P}$ neutral } & \multicolumn{3}{|c|}{$\mathrm{PFJ}+\mathrm{P}$ under } & \multicolumn{3}{|c|}{$\mathrm{PFJ}+\mathrm{P}$ over } \\
\hline & Mean & SD & Mean & SD & & Mean & SD & & Mean & SD & & Mean & SD & \\
\hline 10 & -3.3 & 0.6 & -1.9 & 1.8 & * & 0.4 & 1.8 & ns & -0.6 & 0.9 & ns & -1.0 & 1.0 & ns \\
\hline 20 & -3.6 & 2.5 & -2.1 & 2.4 & *** & 0.4 & 2.0 & ns & -1.0 & 1.1 & * & -1.3 & 1.9 & $* * *$ \\
\hline 30 & -4.8 & 2.7 & -2.8 & 2.6 & $* * *$ & -0.2 & 2.7 & ns & -1.6 & 1.6 & $* * *$ & -2.0 & 2.2 & **** \\
\hline 40 & -4.5 & 2.9 & -2.2 & 2.2 & $* * *$ & 0.0 & 2.1 & ns & -1.2 & 1.1 & $* *$ & -1.6 & 2.0 & **** \\
\hline 50 & -3.5 & 3.2 & -1.4 & 2.4 & $* * *$ & 0.7 & 2.6 & ns & -0.4 & 0.8 & $\mathrm{~ns}$ & -0.5 & 1.7 & ns \\
\hline 60 & -1.8 & 3.4 & -0.4 & 2.5 & ns & 1.5 & 3.0 & $* * *$ & 1.2 & 1.3 & $*$ & 0.8 & 1.8 & ns \\
\hline 70 & 0.4 & 3.8 & 0.3 & 2.3 & ns & 1.9 & 3.7 & $* * *$ & 2.2 & 2.9 & $* * *$ & 1.2 & 2.7 & **** \\
\hline
\end{tabular}

Table 4 Mean (SD) LFCC translation for resisted open chain motions in the native knee and differences in translation in resurfaced conditions compared to the native knee, at different flexion angles

\begin{tabular}{|c|c|c|c|c|c|c|c|c|c|c|c|c|c|c|}
\hline \multirow{3}{*}{$\begin{array}{l}\text { Tibiofemoral } \\
\text { flexion }\end{array}$} & \multirow{2}{*}{\multicolumn{2}{|c|}{ Native opened }} & \multicolumn{12}{|c|}{ Difference wrt. native opened } \\
\hline & & & \multicolumn{3}{|l|}{ PFJ } & \multicolumn{3}{|c|}{ PFJ + P neutral } & \multicolumn{3}{|c|}{$\mathrm{PFJ}+\mathrm{P}$ under } & \multicolumn{3}{|c|}{$\mathrm{PFJ}+\mathrm{P}$ over } \\
\hline & Mean & SD & Mean & SD & & Mean & SD & & Mean & SD & & Mean & SD & \\
\hline 10 & -0.719 & 0.008 & -0.026 & 0.027 & $*$ & -0.016 & 0.020 & ns & -0.027 & 0.006 & $* * *$ & -0.026 & 0.014 & $* * *$ \\
\hline 20 & -0.718 & 0.018 & -0.021 & 0.025 & $* *$ & -0.002 & 0.018 & ns & -0.024 & 0.029 & $* * *$ & -0.028 & 0.027 & $* * *$ \\
\hline 30 & -0.723 & 0.031 & -0.025 & 0.042 & $* * *$ & -0.009 & 0.047 & $\mathrm{~ns}$ & -0.035 & 0.024 & $* * *$ & -0.046 & 0.042 & $* * *$ \\
\hline 40 & -0.690 & 0.023 & -0.012 & 0.028 & $* * *$ & 0.007 & 0.041 & ns & -0.014 & 0.038 & $*$ & -0.023 & 0.052 & $* * *$ \\
\hline 50 & -0.670 & 0.020 & -0.013 & 0.026 & $*$ & 0.009 & 0.041 & ns & -0.005 & 0.018 & ns & -0.005 & 0.030 & ns \\
\hline 60 & -0.657 & 0.014 & -0.011 & 0.032 & ns & 0.001 & 0.040 & $\mathrm{~ns}$ & 0.008 & 0.020 & ns & 0.004 & 0.027 & ns \\
\hline 70 & -0.647 & 0.011 & -0.010 & 0.018 & ns & 0.006 & 0.037 & ns & 0.011 & 0.028 & ns & -0.002 & 0.021 & ns \\
\hline
\end{tabular}



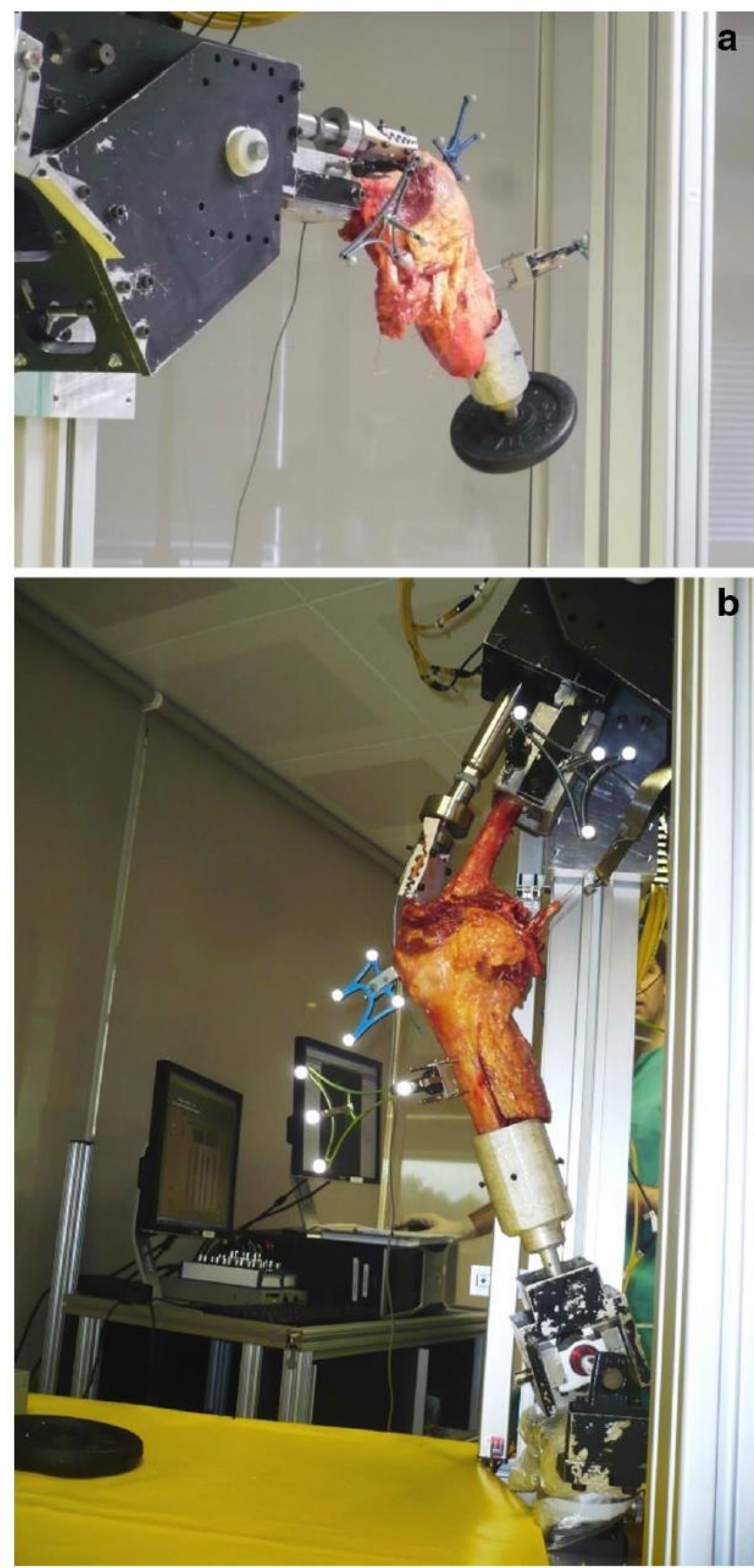

Fig. 1 Open chain movement with 3-kg resistance (a) and squat movement (b) 

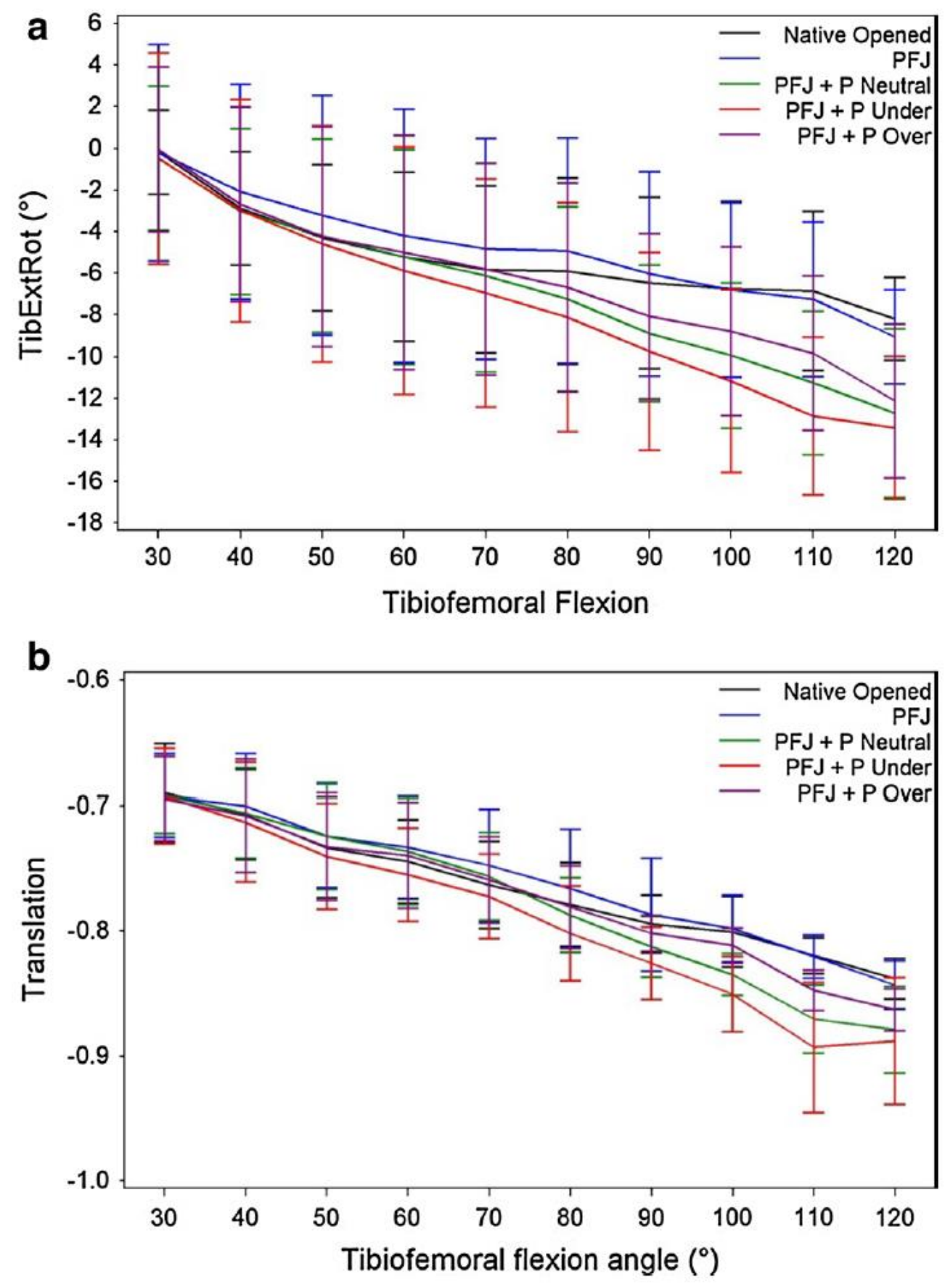

Fig. 2 Average tibial rotation $\left({ }^{\circ}\right)(a)$ and lateral femoral condyle centre translation (fraction of AP size tibia) (b), plotted as function of the tibiofemoral flexion angle $\left({ }^{\circ}\right)$, for squat motions, with the five conditions superimposed. TR tibial rotation (-= internal/+= external), translation translation of LFCC (+= anterior/-= posterior), natural opened natural joint, PFJ isolated trochlear resurfacing, PFJ + P neutral trochlea and patella resurfacing with patella centred on ridge, $\mathrm{PFJ}+\mathrm{P}$ under under-resection or overstuffing patella, $\mathrm{PFJ}+\mathrm{P}$ over over-resection or thinning patella 

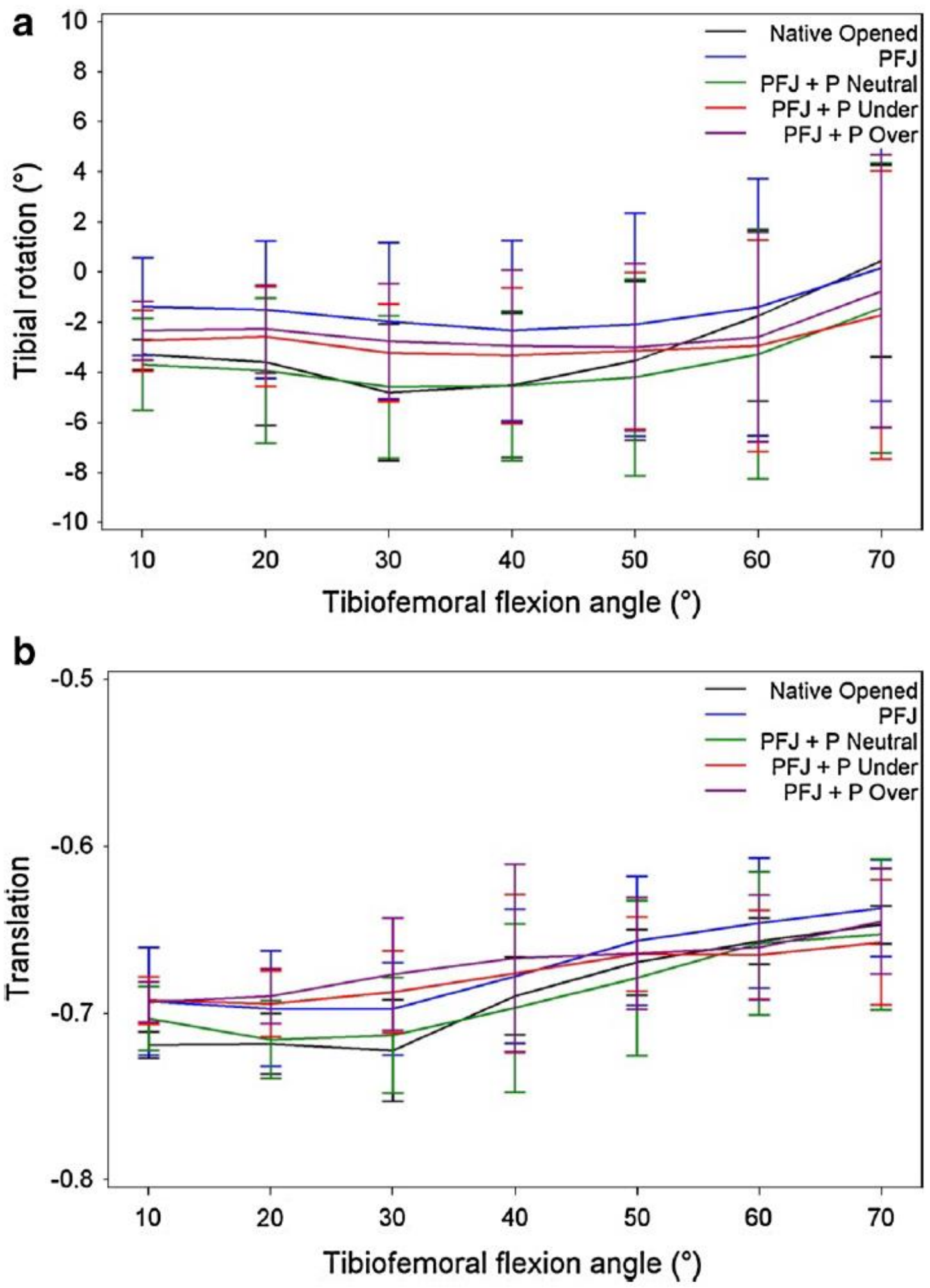

Fig. 3 Average tibial rotation $\left({ }^{\circ}\right)(a)$ and lateral femoral condyle centre translation (fraction of AP size tibia) (b) plotted as function of the tibiofemoral flexion angle $\left({ }^{\circ}\right)$, for open chain motions with 3-kg resistance, with the five conditions superimposed 